\title{
A partition-based approach to identify gene- environment interactions in genome wide association studies
}

\author{
Ruixue Fan ${ }^{1}$, Chien-Hsun Huang ${ }^{1}$, Inchi Hu², Haitian Wang ${ }^{3}$, Tian Zheng ${ }^{1}$, Shaw-Hwa Lo ${ }^{\text {* }}$ \\ From Genetic Analysis Workshop 18 \\ Stevenson, WA, USA. 13-17 October 2012
}

\begin{abstract}
It is believed that almost all common diseases are the consequence of complex interactions between genetic markers and environmental factors. However, few such interactions have been documented to date. Conventional statistical methods for detecting gene and environmental interactions are often based on the linear regression model, which assumes a linear interaction effect. In this study, we propose a nonparametric partition-based approach that is able to capture complex interaction patterns. We apply this method to the real data set of hypertension provided by Genetic Analysis Workshop 18. Compared with the linear regression model, the proposed approach is able to identify many additional variants with significant gene-environmental interaction effects. We further investigate one single-nucleotide polymorphism identified by our method and show that its gene-environmental interaction effect is, indeed, nonlinear. To adjust for the family dependence of phenotypes, we apply different permutation strategies and investigate their effects on the outcomes.
\end{abstract}

\section{Background}

Genome-wide association studies (GWAS) have successfully discovered many common variants associated with complex diseases, but the single-nucleotide polymorphisms (SNPs) identified so far account for a small proportion of the total heritability in quantitative traits [1]. Increasing evidence shows that gene-environment $(\mathrm{G} \times \mathrm{E})$ interactions are widely involved in the etiology of complex diseases, including diabetes, cancer, and psychiatric disorders [2,3]. The investigation of $\mathrm{G} \times \mathrm{E}$ interactions will not only facilitate the identification of novel genes whose marginal effects are undetectable, but also provide insights into disease etiology and hence greatly benefit drug development and personalized therapy.

The commonly applied methods to detect G×E interactions are based on linear or logistic regression models [4]. In particular, for quantitative outcomes, a linear model is considered in the form of

\footnotetext{
* Correspondence: slo@stat.columbia.edu

'Department of Statistics, Columbia University, 1255 Amsterdam Avenue, 10th Floor, New York, NY 10027, USA

Full list of author information is available at the end of the article
}

$$
y=\beta_{0}+\beta_{1} G+\beta_{2} E+\beta_{3} G \times E+\varepsilon
$$

where $G$ is the genotype of a SNP, $E$ is the environmental factor, $\varepsilon$ is a normally distributed random error, and $\beta_{3}$ is the coefficient corresponding to the interaction term. If $\beta_{3}=0$, the conditional effect of the SNP is constant across different levels of the environmental factor and we conclude that there is no $\mathrm{G} \times \mathrm{E}$ interaction. This model assumes a linear interaction effect; given $G$, the outcome $y$ is linearly related with $E$. However, in practice, it is likely that the interaction schemes are more complicated so that the linear model will probably fail to capture the interaction effect. Therefore, there is a pressing need to develop novel statistical approaches for genome-wide $\mathrm{G} \times \mathrm{E}$ interaction studies. Here we propose a nonparametric partition-based approach to detect $\mathrm{G} \times \mathrm{E}$ interactions and conduct a GWAS for hypertension using the real data set provided by Genetic Analysis Workshop 18 (GAW18). For each SNP, both the linear regression model and the proposed method are used to evaluate its interaction effect with each of the 4 environmental factors: age, gender, smoking status, and medicine. We note that, compared with the linear model, the 
proposed method is able to identify many additional SNPs. We further study the interaction pattern between SNP rs17206492 and medicine, and find that this interaction effect is, indeed, nonlinear. We also investigate different permutation strategies in the presence or absence of pedigree dependence of the phenotype.

\section{Methods}

\section{Data set}

The GAW18 data set consists of GWAS data and whole genome sequence data with longitudinal phenotypes for hypertension and related traits from Type 2 Diabetes Genetic Exploration by Next-generation sequencing in Ethnic Samples (T2D-GENES) Project 2. There are 939 individuals in total, and we include in our analysis only the 849 individuals with both phenotype data and imputed sequence information. Each individual has measurements for up to 4 time points. At each visit, systolic blood pressure (SBP) and diastolic blood pressure (DBP) were measured; covariates including age, use of antihypertensive medication, and current tobacco smoking status were also recorded. Gender and pedigree are known for each subject. Genotypes of odd-numbered chromosomes are provided. In our study, we focused on chromosome 3 as suggested by the workshop organizer for the sake of comparison. Although we had access to the answers for the simulated data set, we used only the real data set in our analysis.

\section{A general framework-a partition-based association measure}

Suppose there are $n$ independent subjects that can be separated by a partition $\prod$. An association measure between the outcome $Y$ and the partition $\prod$ is defined as:

$$
I=\sum_{\Pi_{i}} \frac{n_{i}}{n} \frac{\left(\overline{Y_{i}}-\bar{Y}\right)^{2}}{s_{y^{2}}{ }^{2} n_{i}}
$$

where $n_{i}$ is the number of subjects in partition $i, \bar{Y}_{i}$ is the average of the outcome $Y$ for subjects in partition $i$, and $\bar{Y}$ and $s_{y}^{2}$ are the mean and variance of $Y$ from all subjects. It has been shown that under the null hypothesis $\prod$ does not have influence on $Y, I$ asymptotically converges to a weighted sum of $\chi^{2}{ }_{1}$ distributions [5]. It has higher power than linear regression or logistic regression models, even in sparse partitions.

\section{GXE association measure I}

Consider a marker $G$ and an environmental factor $E$. Suppose $G$ has 3 phenotypes, AA, Aa, and aa (A refers to the major allele and a the minor allele), coded as 0 , 1 , and 2. Suppose $E$ is divided into 3 categories: 0,1 , and 2 . Hereby $G$ and $E$ together create 9 partitions for
Table 1 Partitions created by genotypic and environmental factors

\begin{tabular}{ccccc}
\hline & $\boldsymbol{E}=\mathbf{0}$ & $\boldsymbol{E}=\mathbf{1}$ & $\boldsymbol{E}=\mathbf{2}$ & Total \\
\hline$G=0$ & $n_{00}$ & $n_{01}$ & $n_{02}$ & $n_{0}$ \\
$G=1$ & $n_{10}$ & $n_{11}$ & $n_{12}$ & $n_{1 .}$ \\
$G=2$ & $n_{20}$ & $n_{21}$ & $n_{22}$ & $n_{2}$. \\
Total & $n_{0}$ & $n_{\cdot 1}$ & $n_{\cdot 2}$ & $n_{.}$ \\
\hline
\end{tabular}

n.., Total number of subjects, $n_{i j}$, number of subjects in partition $i j, n_{i,}$ number of subjects in group $G=i ; n_{. j}$, number of subjects in group $E=j$.

all subjects (Table 1). From the general framework in the last section, an association measure that evaluates the total effect of $G$ and $E$ on the phenotype is:

$$
I_{T}=\sum_{i=1}^{3} \sum_{j=1}^{3} \frac{n_{i j}}{n_{. .}} \cdot \frac{\left(\bar{y}_{i j}-\bar{y}\right)^{2}}{s^{2} / n_{i j}}
$$

where all the terms are similarly defined as before and $y$ denotes the phenotype. The marginal effects of $G$ and $E$ can be obtained in a similar fashion:

$$
I_{G}=\sum_{i=1}^{3} \frac{n_{i \bullet}}{n_{. .}} \cdot \frac{\left(\bar{y}_{i \bullet}-\bar{\gamma}\right)^{2}}{s^{2}{ }_{\gamma} / n_{i \bullet}} ; I_{E}=\sum_{j=1}^{3} \frac{n_{\bullet j}}{n_{. .}} \cdot \frac{\left(\bar{y}_{\bullet j}-\bar{\gamma}\right)^{2}}{s^{2}{ }_{\gamma} / n_{\bullet j}}
$$

The test statistic that measures the $\mathrm{G} \times \mathrm{E}$ interaction effect is defined as the difference between the total effect and the maximum of the two marginal effects:

$$
I_{G \times E}=I_{T}-\max \left(I_{G}, I_{E}\right)
$$

The significance of $I_{G \times E}$ is evaluated by the method of permutation.

\section{Permutation strategies}

We consider 3 permutation strategies in our analysis: global permutation, local permutation, and residual permutation. Let $y_{i j}$ denote the phenotype of the $j^{\text {th }}$ individual in the $i^{\text {th }}$ pedigree. Global permutation is to permute phenotypes over all individuals. For local permutation, the phenotypes are permuted within each pedigree. In residual permutation, we first compute the residuals for each individual $e_{i j}=y_{i j}-\bar{y}_{i}$, where $\bar{y}_{i \text {. }}$ is the average phenotype for pedigree $i$, then permute $e_{i j}$ over all subjects to obtain a permuted residual $e_{i j}^{*}$ for each individual. The permuted $Y$ values $\gamma_{i j}^{*}$ are obtained by $\gamma_{i j}^{*}=\bar{\gamma}_{i .}+e_{i j}^{*}$. Both local permutation and residual permutation assume $\gamma_{i j}=\bar{y}_{i .}+\varepsilon_{i j}$, where $E\left(\varepsilon_{i j}\right)=0$ and $\left\{\varepsilon_{i j}\right\}$ are independent. Residual permutation further assumes that $\left\{\varepsilon_{i j}\right\}$ have the same distribution.

\section{Results}

\section{Partitions created by environmental factors}

The real data set from GAW18 contains the records of 4 environmental factors: age, gender, smoking status, 
and antihypertensive medication usage (medicine). Because gender is a binary variable, it partitions all individuals into 2 groups. Although this data set provides longitudinal measurements of age, smoking, and medicine, the records have many missing values (only 187 subjects have complete measurements for all 4 visits). Therefore, for each individual, we summarized these covariates by either the averaged value (for age) or the sum (for smoking and medicine) across different time points from available records and used these summarized quantities in our analysis. Similarly, averaged SBP and averaged DBP were considered as outcomes. Here we created 3 partitions by each of age, smoking, and medicine (Table 2).

\section{SNPs with significant GXE interaction effects}

In the GWAS data set provided by GAW18, there are 62,915 SNPs on chromosome 3. For each SNP, we evaluated its interaction effect with each of the 4 environmental factors on both SBP and DBP using the linear regression model (LRM) and the proposed partition-based score $I$ (PBI). $p$ Values of LRM were derived from the asymptotic distribution of the regression coefficient $\beta_{3}$ and $p$ values of $P B I$ were computed from $10^{7}$ permutations using global, local, or residual permutation procedures. Table 3 lists the number of SNPs with $p$ values less than the Bonferroni-corrected significance level $\left(7.9^{*} 10^{-7}\right)$ for all interactions under consideration. Compared with LRM, PBI identified many additional significant SNPs, especially when testing the $\mathrm{G} \times \mathrm{E}$ interaction effects with medicine. The reason, we believe, is that the interaction modeled by LRM is restricted to the linear form, whereas $P B I$ is able to capture nonlinear and complicated interaction patterns. To confirm our hypothesis, we further analyzed the SNP rs17206492, which was identified by $P B I$ (using any of the 3 permutation strategies) to have strong G×Medicine interaction effect on DBP, but was not selected by LRM. The left panel of Figure 1 shows that the averaged values of DBP in individuals not carrying the minor allele (genotype 0 ) and in individuals carrying the minor allele (genotype 1) are almost the same, indicating that rs17206492 does not have strong marginal effect. However, with the increase of medication usage, when the genotype is 1 (middle panel of Figure 1), DBP first decreases and then increases; but when the genotype is 0 (right panel of Figure 1), DBP first increases and then decreases. This nonlinear interaction scheme cannot be detected by LRM, but is captured by our model-free test statistic $P B I$.

\section{Effect of different permutation strategies}

There are 20 pedigrees in the GAW18 data set. Both the analysis of variance (ANOVA) test and the nonparametric Kruskal-Wallis test indicate that the mean DBP values of different pedigrees are different, whereas the mean SBP values are the same (Table 4). When evaluating the $p$ values of $P B I$, we performed 3 types of permutation: global (GP), local (LP), and residual (RP) permutations. Both LP and RP adjust for familial relatedness between individuals. For SBP, except for the environmental factor age, the results from 3 permutation methods coincide substantially (see Table 3 and Figure 2), which is consistent with the conclusion from ANOVA and Kruskal-Wallis test. In contrast, for DBP, the results of GP are quite different from the results of LP or RP, especially when assessing the interaction effect with medicine (see Table 3 and Figure 2). In this situation, the results from LP or RP are more reliable because they take into account the family dependence

Table 2 Partitions based on the summarized quantities of age, smoking status, or medicine

\begin{tabular}{ccc}
\hline By age* & By smoking & By medicine \\
\hline $16 \sim 33.44 \rightarrow$ Partition 0 & $0 \rightarrow$ Partition 0 & $0 \rightarrow$ Partition 0 \\
33.45 50.30 $\rightarrow$ Partition 1 & $1 \rightarrow$ Partition 1 & $1 \rightarrow$ Partition 1 \\
$50.31 \sim 94.20 \rightarrow$ Partition 2 & $2,3,4 \rightarrow$ Partition 2 & $2,3,4 \rightarrow$ Partition 2 \\
\hline
\end{tabular}

${ }^{*}$ The age group is divided by the $33 \%$ quantile (33.44) and 67\% quantile (50.30). The minimum age is 16 and the maximum age is 94.2.

Table 3 Number of significant SNPs with $p$ value less than $7.9 * 10^{-7} *$

\begin{tabular}{|c|c|c|c|c|c|c|c|c|}
\hline \multirow[t]{2}{*}{ Environmental factor } & \multicolumn{4}{|c|}{ DBP } & \multicolumn{4}{|c|}{ SBP } \\
\hline & LRM & $\begin{array}{l}P B I \\
(G P)\end{array}$ & $\begin{array}{l}P B I \\
(L P)\end{array}$ & $\begin{array}{l}P B I \\
(R P)\end{array}$ & LRM & $\begin{array}{l}P B I \\
(G P)\end{array}$ & $\begin{array}{l}P B I \\
(L P)\end{array}$ & $\begin{array}{c}P B I \\
(R P)\end{array}$ \\
\hline Age & 0 & 4 & 7 & 3 & 6 & 16 & 33 & 20 \\
\hline Smoke & 0 & 6 & 3 & 3 & 0 & 0 & 0 & 0 \\
\hline Gender & 0 & 42 & 37 & 36 & 0 & 1 & 1 & 1 \\
\hline Medicine & 4 & 80 & 53 & 33 & 1 & 65 & 65 & 57 \\
\hline
\end{tabular}

$G P$, Global permutation; $L P$, local permutation; $L R M$, linear regression model; $P B I$, partition-based $l ; R P$, residual permutation.

${ }^{*} 7.9^{*} 10^{-7}$ is the Bonferroni corrected $p$ value. 


\section{SNP: rs17206492}
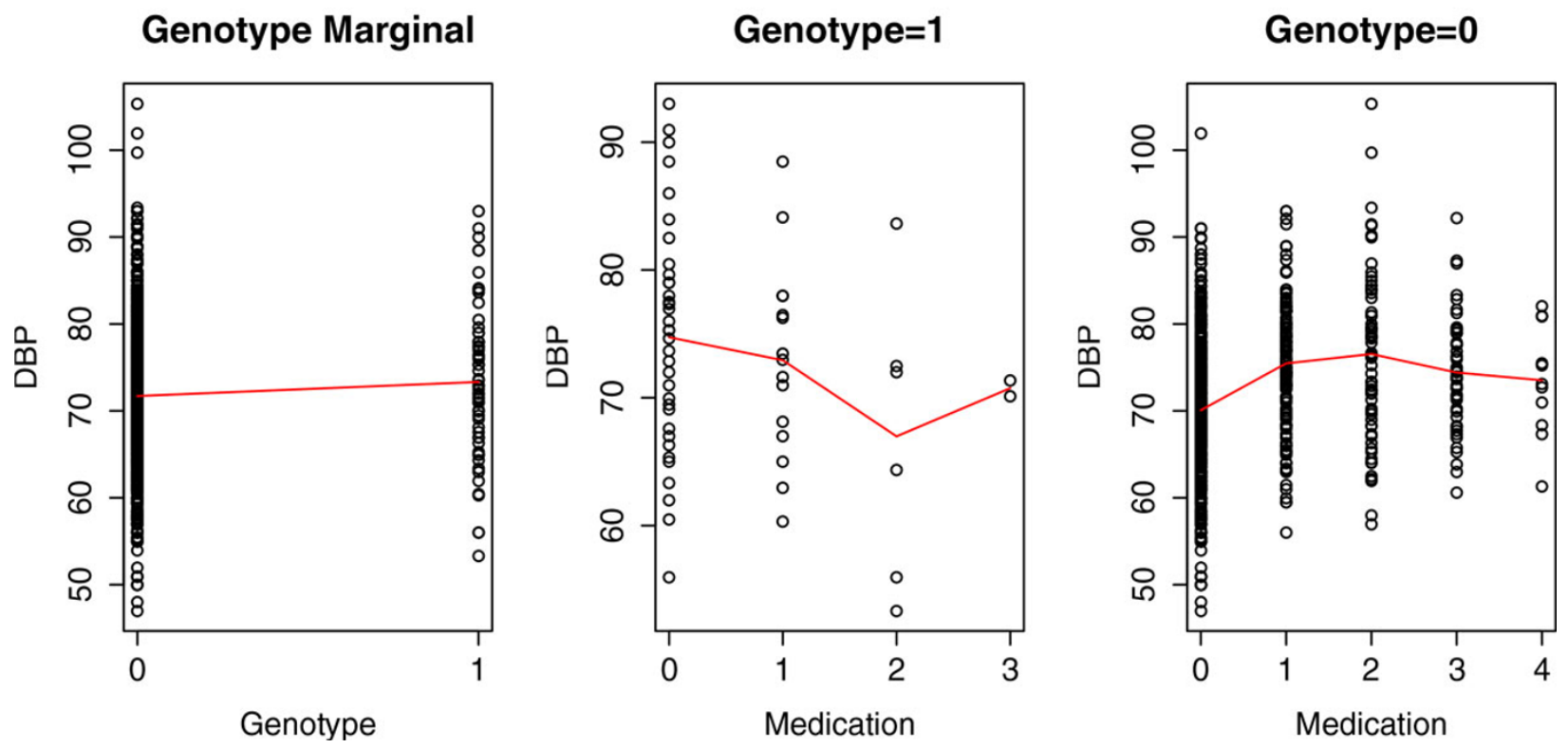

Figure $1 \mathrm{GXE}$ interaction effect of SNP $r$ s 17206492 and medicine. The marginal effect of the genotype (left), the medication effect when genotype $=1$ (middle), and the medication effect when genotype is 0 (right).

of the phenotype. In addition, LP tends to select more markers than RP; this may be because the data violate the assumption that $\left\{\varepsilon_{i j}\right\}$ have the same distribution. Moreover, SNPs identified by LP and RP overlap considerably and the consistency of results from these two permutation strategies can be an indicator of true signal.

\section{Discussion}

In this paper, we have proposed a partition-based approach $P B I$ to detect $\mathrm{G} \times \mathrm{E}$ interactions, which is nonparametric and model-free. The test statistic is derived from a partition-based measure $I$, and the interaction information score $I_{G \times E}$ is defined as the difference between the total score $I_{T}$ and the maximum of the marginal scores. Intuitively, if the genetic and the environmental factors have strong interaction effect, $I_{T}$ will be far greater than both marginal scores; hence $I_{G \times E}$ will be positive and large. If not, $I_{T}$ will be no greater than at least 1 of the marginal scores. Therefore, $I_{G \times E}$ evaluates the amount of influence of the $\mathrm{G} \times \mathrm{E}$ interactions on the phenotype.

Table $4 p$ Values for testing the pedigree dependence of SBP and DBP

\begin{tabular}{ccc}
\hline & ANOVA test & Kruskal-Wallis test \\
\hline SBP & 0.155 & 0.433 \\
DBP & 0.000625 & 0.0004226 \\
\hline
\end{tabular}

When applied to the real data set about hypertension provided by GAW18, PBI identified many more markers than the traditional linear regression method. Because our approach is model-free, it is able to capture complicated interaction patterns that are difficult to detect in linear model. The significance of $I_{G \times E}$ is evaluated by permutation. LP and RP adjust effectively for the family dependence of the phenotype. Despite the fact that the proposed procedure selects more SNPs than linear regression, there is very little experimental evidence of $\mathrm{G} \times \mathrm{E}$ interactions for hypertension in the current literature to verify our findings. Therefore, biological studies will be required to investigate our results. Modifications of $P B I$ have successfully identified gene-gene interactions and constructed genetic networks for breast cancer [6] and rheumatoid arthritis [7]. Moreover, $P B I$ can be extended to evaluate the interaction effects between rare variants and environmental factors. Because of the low frequencies of rare variants $(<1 \%)$, we can apply a genebased approach by collapsing rare variants in a gene [8-11] and creating partitions based on the collapsed information.

Competing interests

The authors declare that they have no competing interests.

Authors' contributions

SHL and RF designed the study. RF, CHH and SHL performed the study. RF, $\mathrm{CHH}, \mathrm{IH}, \mathrm{HW}, \mathrm{TZ}$ and $\mathrm{SHL}$ contributed to analysis of the data. RF and $\mathrm{SHL}$ drafted the manuscript. All authors read and approved the final manuscript. 


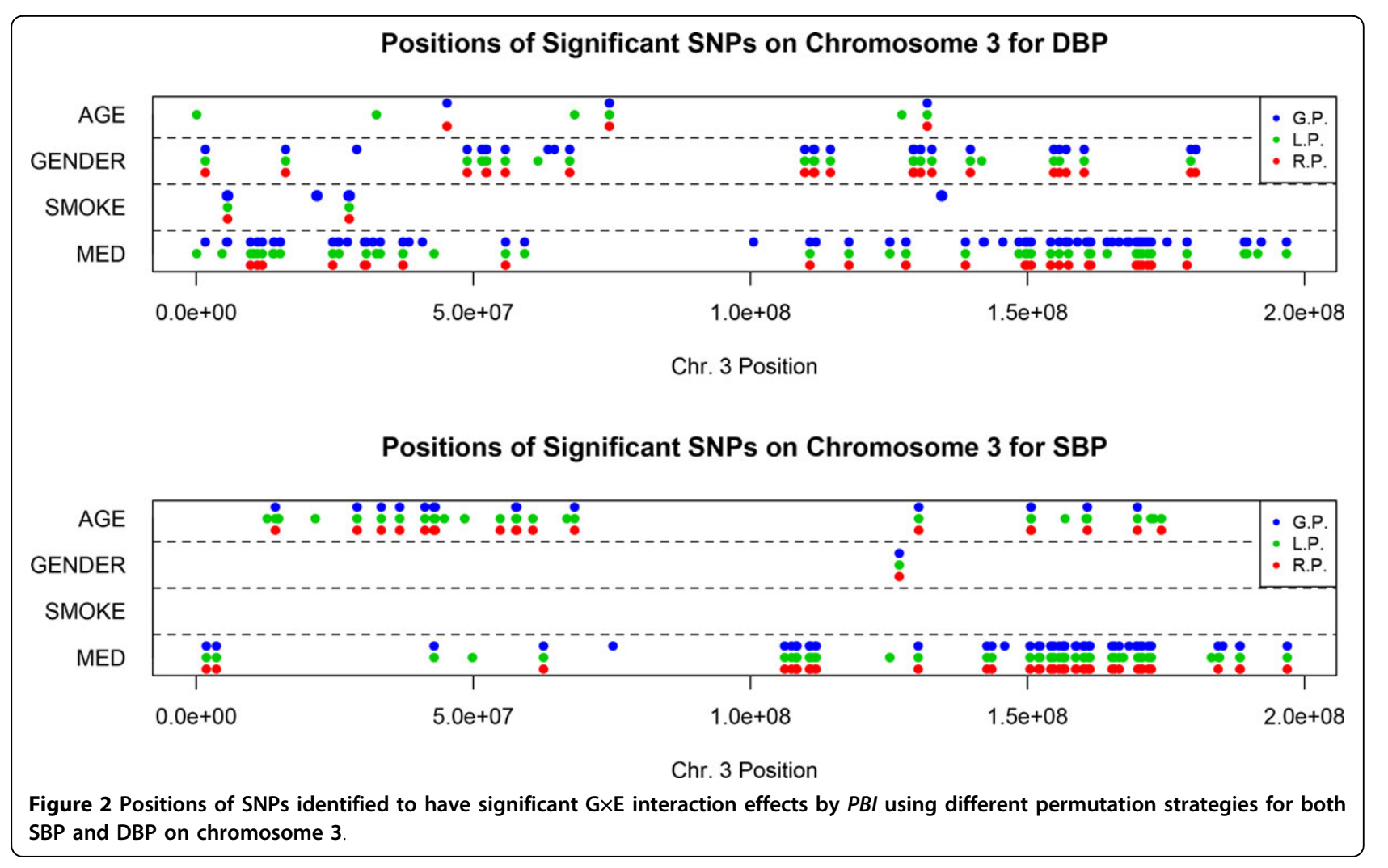

\section{Acknowledgements}

This research is supported by National Institutes of Health Grant R01 GM070789, GM070789-0551 and by Hong Kong Research Grant Council (642207 and 601312).

The GAW18 whole genome sequence data were provided by the T2DGENES Consortium, which is supported by NIH grants U01 DK085524, U01 DK085584, U01 DK085501, U01 DK085526, and U01 DK085545. The other genetic and phenotypic data for GAW18 were provided by the San Antonio Family Heart Study and San Antonio Family Diabetes/Gallbladder Study, which are supported by NIH grants P01 HL045222, R01 DK047482, and R01 DK053889. The Genetic Analysis Workshop is supported by NIH grant R01 GM031575.

This article has been published as part of BMC Proceedings Volume 8 Supplement 1, 2014: Genetic Analysis Workshop 18. The full contents of the supplement are available online at http://www.biomedcentral.com/bmcproc/ supplements/8/S1. Publication charges for this supplement were funded by the Texas Biomedical Research Institute.

\section{Authors' details}

'Department of Statistics, Columbia University, 1255 Amsterdam Avenue, 10th Floor, New York, NY 10027, USA. ${ }^{2}$ ISOM, Hong Kong University of Science and Technology, Hong Kong. ${ }^{3}$ Division of Biostatistics, School of Public Health and Primary Care, The Chinese University of Hong Kong, Hong Kong.

Published: 17 June 2014

\section{References}

1. Manolio TA, Collins FS, Cox NJ, Goldstein DB, Hindorff LA, Hunter DJ, McCarthy MI, Ramos EM, Cardon LR, Chakravarti A, et al: Finding the missing heritability of complex diseases. Nature 2009, 461:747-753.

2. Andreasen $\mathrm{CH}$, Mogensen MS, Borch-Johnsen $\mathrm{K}$, Sandbæk A, Lauritzen T, Sorensen TI, Hansen L, Almind K, Jorgensen T, Pedersen O, et al: Nonreplication of genome-wide based associations between common variants in INSIG2 and PFKP and obesity in studies of 18,014 Danes. PLoS One 2008, 3:e2872.
3. Hamza TH, Chen H, Hill-Burns EM, Rhodes SL, Montimurro J, Kay DM, Tenesa A, Kusel VI, Sheehan P, Eaaswarkhanth M, et al: Genome-wide gene-environment study identifies glutamate receptor gene GRIN2A as a Parkinson's disease modifier gene via interaction with coffee. PLoS Genetics 2011, 7:e1002237.

4. Kraft P, Yen YC, Stram DO, Morrison J, Gauderman WJ: Exploiting geneenvironment interaction to detect genetic associations. Hum Hered 2007, 63:111-119.

5. Chernoff H, Lo SH, Zheng T: Discovering influential variables: a method of partitions. Ann Appl Stat 2009, 3:1335-1369.

6. Lo SH, Zheng T: A demonstration and findings of a statistical approach through reanalysis of inflammatory bowel disease data. Proc Natl Acad Sci U S A 2004, 101:10386-10391.

7. Huang CH, Cong L, Xie J, Qiao B, Lo SH, Zheng T: Rheumatoid arthritisassociated gene-gene interaction network for rheumatoid arthritis candidate genes. BMC Proc 2009, 3(suppl 7):S75.

8. Dering C, Pugh E, Ziegler A: Statistical analysis of rare sequence variants an overview of collapsing methods. Genet Epidemiol 2011, 35:S12-S17.

9. Fan R, Huang CH, Lo SH, Zheng T, lonita-Laza I: Identifying rare disease variants in the Genetic Analysis Workshop 17 simulated data: a comparison of several statistical approaches. BMC Proc 2011, 5:S17.

10. Chen G, Wei P, DeStefano AL: Incorporating biological information into association studies of sequencing data. Genet Epidemiol 2011, 35:S29-S34.

11. Wu MC, Lee S, Cai T, Li Y, Boehnke M, Lin X: Rare-variant association testing for sequencing data with the sequence kernel association test. Am J Hum Genet 2011, 89:82-93.

doi:10.1186/1753-6561-8-S1-S60

Cite this article as: Fan et al:: A partition-based approach to identify gene-environment interactions in genome wide association studies. BMC Proceedings 2014 8(Suppl 1):S60. 